\title{
Transition metal $d$-band occupancy in skutterudites studied by electron energy-loss spectroscopy
}

\author{
$\varnothing$. Prytz \\ Centre for Materials Science and Nanotechnology, University of Oslo, P.O. Box 1126 Blindern, N-0318 Oslo, Norway \\ J. Taftø \\ Department of Physics, University of Oslo, P.O. Box 1048 Blindern, N-0316 Oslo, Norway \\ C. C. Ahn and B. Fultz \\ Division of Engineering and Applied Science, California Institute of Technology, M/C 138-78, Pasadena, California 91125, USA
}

(Received 18 August 2006; revised manuscript received 28 November 2006; published 13 March 2007)

\begin{abstract}
The transition-metal $3 d$ occupancy of a series of thermoelectric skutterudites is investigated using electron energy-loss spectroscopy. We find that bonding causes an emptying of the $3 d$ states in the binary skutterudites $\mathrm{CoP}_{3}, \mathrm{CoAs}_{3}, \mathrm{CoSb}_{3}$, and $\mathrm{NiP}_{3}$, while compared to the pure Fe the $3 d$ occupancy in $\mathrm{LaFe}_{4} \mathrm{P}_{12}$ is significantly increased, consistent with the idea that each interstitial La atom (rattler) donates three electrons to compensate for missing valence electron of $\mathrm{Fe}$ as compared to $\mathrm{Co}$. These experimental results are in agreement with previous models suggesting a predominantly covalent bonding between transition metal and pnictogen atoms in skutterudites, and provide evidence of charge transfer from La to the Fe-P complex in $\mathrm{LaFe}_{4} \mathrm{P}_{12}$.
\end{abstract}

DOI: 10.1103/PhysRevB.75.125109

PACS number(s): 79.20.Uv, 71.20.Be, 72.20.Pa

\section{INTRODUCTION}

In the search for new and more effective thermoelectric materials, the class of materials with the so-called skutterudite crystal structure (space group $\operatorname{Im} \overline{3}$ ) has received much attention. These materials have the formula unit $\mathrm{TMPn}_{3}$, where TM is transition metal, usually one of the column 9 elements $\mathrm{Co}, \mathrm{Rh}$, or Ir, while Pn is one of the pnictogens $\mathrm{P}$, $\mathrm{As}$, or $\mathrm{Sb}$. The cubic unit cell of the skutterudites is made up of eight smaller primitive cubes of the transition metal, six of which are filled by rectangular four-member rings of the pnictogen. ${ }^{1}$ This leaves two rather large voids in the center of the two remaining metal cubes.

The skutterudites are especially interesting due to their tolerance for modification using other atomic species while retaining their crystal structure. For example, filling of the voids with heavy atomic species (called "rattlers") has been observed to lead to dramatic reductions in the thermal conductivity of the material, which is beneficial for the thermoelectric properties. Furthermore, substitution on the transition metal or pnictogen sites can control the electronic properties. A virtual continuum of modified skutterudites may be formed, with compositions such as $\mathrm{Ln}_{y} \mathrm{TM}_{4-x} \mathrm{Fe}_{x} \mathrm{Pn}_{12}$. Here, $\mathrm{Ln}$ is one of the lanthanides, often $\mathrm{La}$ or $\mathrm{Ce}, \mathrm{TM}$ is one of the column 9 transition metals as above, $\mathrm{Pn}$ is one of the pnictogens, and a column 8 transition metal such as $\mathrm{Fe}$ is introduced to maintain charge balance. ${ }^{2}$ Tailoring their electronic and thermal properties is therefore possible, and modified skutterudite materials are indeed among the most promising of a new generation of thermoelectric materials. ${ }^{2-4}$ Several theoretical $^{5-8}$ and experimental $^{9-13}$ studies of the electronic structure of Cobased skutterudites have been performed. A particular interesting signature of the bonding and electronic structure is the occupancy of the $3 d$ states of Co. Anno et al. ${ }^{12}$ used x-ray photoelectron spectroscopy (XPS) to study the occupied states of $\mathrm{CoAs}_{3}$ and $\mathrm{CoSb}_{3}$. They found evidence of a small charge transfer from metal to pnictogen atoms and suggest a hybridization between the metal $d$ and pnictogen $p$ states. In this study, we use the complementary technique of electron energy-loss spectroscopy (EELS) to investigate the effects of bonding by probing the density of empty transition metal $3 d$ states above the Fermi level of $\mathrm{CoP}_{3}, \mathrm{CoAs}_{3}$, and $\mathrm{CoSb}_{3}$, as well as $\mathrm{NiP}_{3}$ and the filled skutterudite $\mathrm{LaFe}_{4} \mathrm{P}_{12}$. The EELS analyses are done in a probe forming transmission electron microscope (TEM) with $200 \mathrm{keV}$ incident electrons. The ability to form a small probe with a TEM means that EELS can be used on small volumes of materials.

Electron energy-loss spectra are obtained by analyzing the energy distribution of electrons transmitted through the sample. Some of these electrons will have lost energy through inelastic scattering in the sample, for example, by exciting core electrons from their ground state. These excitations involve transitions of electrons from the occupied core levels into empty states above the Fermi level. The probability of a transition occurring is dependent on the spatial overlap of the initial and final states, effectively restricting the transitions to final states centered on the same atom as the initial states. Since the initial core levels are highly localized in energy, these transitions are mainly sensitive to final-state effects. The occupancy of these final states may change due to bonding through charge transfer or hybridization, and this will be reflected in the EEL spectrum. Thus, using EELS, we probe the local density of empty states of the material, potentially revealing a wealth of information on both bonding and crystal structure. ${ }^{14-18}$

The spectra obtained in this study include sharp energyloss features associated with dipole transitions of the type TM $2 p_{1 / 2} \rightarrow 3 d_{3 / 2}\left(L_{2}\right)$ and $2 p_{3 / 2} \rightarrow 3 d_{5 / 2,3 / 2}\left(L_{3}\right)$. In the first row transition metals, these excitations take place below $1000 \mathrm{eV}$, an energy range well suited for EELS studies. In a single electron approximation, the intensity of these features is then given by

$$
I \propto \rho^{u}(3 d)\left|\left\langle 3 d\left|e^{i \Delta \mathbf{k} \cdot \mathbf{r}}\right| 2 p\right\rangle\right|^{2} .
$$


Here, $\rho^{u}(3 d)$ is the unoccupied density of states with transition metal $3 d$ symmetry, $\Delta \mathbf{k}$ is the momentum transfer from the fast electron to the core electron, and $\mathbf{r}$ is the position of the electron with the atom core as the origin.

Previous studies of transition metals ${ }^{19,20}$ and their oxide ${ }^{21}$ have revealed a linear relationship between the intensity of the energy-loss features associated with these transitions (called "white lines") and the $3 d$-band occupancy. This can be used to quantitatively determine the occupancy of the $d$ states. The outer $3 d$ electrons may participate in bonding through charge transfer and hybridization with wave functions from neighboring atoms. Electron energy-loss experiments probe the part of the molecular orbital which is projected onto the atom with the original $2 p$ state. Although one may not be able to distinguish between the effects of covalent or ionic bonding, ${ }^{22}$ important information on bonding, charge compensation, and hybridization upon compound formation can still be revealed. ${ }^{16,23,24}$

The cobalt-based skutterudites are among those which have received most attention in studies of skutterudites as thermoelectric materials. Using EELS, we explore the local electronic structure of cobalt in $\mathrm{CoP}_{3}, \mathrm{CoAs}_{3}$, and $\mathrm{CoSb}_{3}$, thereby investigating the bonding of cobalt in different atomic environments. Furthermore, we study $\mathrm{NiP}_{3}$, a material which is unique in that it forms the skutterudite structure with a noncolumn 9 transition metal.

Finally, we investigate the $d$-band occupancy of Fe in the filled skutterudite $\mathrm{LaFe}_{4} \mathrm{P}_{12}$. Binary skutterudites do not form with column 8 transition metals such as iron, as these lack the outer $3 d$ electrons needed to form the bonds that stabilize the structure. However, when the so-called rattlers such as La or $\mathrm{Ce}$ are introduced in crystal, Fe can be fully substituted onto the Co sites. Thus, we wish to probe the local electronic structure of iron in this material in order to reveal the mechanisms by which this material forms.

The spectra obtained from these materials are compared to those of the pure metals, and the results are discussed in terms of the bonding mechanisms of the skutterudites.

\section{EXPERIMENTAL PROCEDURES AND DATA ANALYSIS}

Samples of $\mathrm{CoP}_{3}$ and $\mathrm{LaFe}_{4} \mathrm{P}_{12}$ were synthesized using a tin-flux technique, ${ }^{25}$ while the $\mathrm{CoAs}_{3}, \mathrm{CoSb}_{3}$, and $\mathrm{NiP}_{3}$ were synthesized by direct reaction of the constituent elements in sealed and evacuated silica tubes. Thin areas for TEM studies were obtained by crushing the samples in an agate mortar and then deposited on a carbon film suspended on a copper grid.

Electron transparent thin films of pure $\mathrm{Fe}$ and $\mathrm{Ni}$ were prepared by thermal evaporation onto single-crystal rocksalt substrates. These films were then floated off the substrates in water and picked up with copper TEM grids. The cobalt sample was prepared by mechanical grinding and subsequent electrochemical polishing using a mixture of $95 \%$ methanol and 5\% perchloric acid as an electrolyte.

The samples were studied in diffraction coupling mode in a Philips EM 430 TEM operated at $200 \mathrm{kV}$, fitted with a Gatan 666 parallel-detection electron energy-loss spectrometer. The spectrometer collects data only in 1022 channels

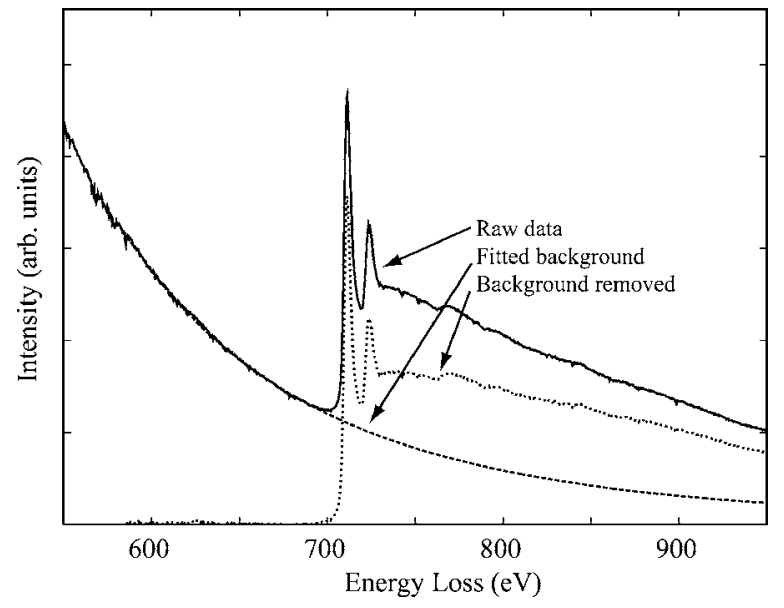

FIG. 1. Example of how a fitted power-law model was used to remove the background contribution at the $\mathrm{Fe} L_{2,3}$ edge.

simultaneously, thus limiting the energy range of each spectrum at high dispersion settings. Low-loss and core-loss spectra were therefore obtained separately. Between five and ten pairs of spectra were obtained at several different locations on each sample.

The composition of all the samples was verified using energy dispersive $\mathrm{x}$-ray analysis, while the crystal structure was checked using electron diffraction. Furthermore, the samples were investigated for oxidation using the oxygen $K$ edge in the EEL spectra. No significant traces of oxygen were found.

The spectra were corrected for the spectrometer dark count, and each spectrum was divided by the spectrometer response function, obtained by uniformly illuminating the detector with electrons. The spectrometer was set to a $0.5 \mathrm{eV}$ dispersion, and each spectrum was obtained as shifted $1.5 \mathrm{eV}$ from the previous spectrum to average out gain variations in the photodiode array. The energy resolution of these experiments was approximately $1.5-2.0 \mathrm{eV}$, as determined by the full width at half maximum of the zero-loss peak. The background below the core loss edge was removed using a power-law model of the form $A e^{-B E}$ fitted to a $30 \mathrm{eV}$ window of the pre-edge intensity, ${ }^{26}$ as seen in Fig. 1. Furthermore, the spectra were deconvolved using the Fourier-ratio method $^{26}$ with the obtained low-loss spectra as input. The spectra were then aligned and summed. No attempt was made to measure any chemical shift in the onset of the transition metal white lines.

The white-line intensity was obtained by integrating the number of counts over a region which for the cobalt containing samples extended typically $26.5 \mathrm{eV}$ beyond the edge onset. For the samples containing $\mathrm{Fe}$ and $\mathrm{Ni}$, the white-line integration regions were approximately 22 and $28 \mathrm{eV}$, respectively. The contribution of the $2 p \rightarrow$ continuum transitions was estimated by a linear function extending from the edge onset to the start of the postedge continuum. This contribution was then subtracted from the edge integral. The resulting intensity was then normalized to a $50 \mathrm{eV}$ window $50 \mathrm{eV}$ beyond the edge onset (see Graetz et al. for details). ${ }^{21}$

In the dipole approximation, only transitions resulting in a change of the angular momentum quantum number of $\Delta l$ 
$= \pm 1$ are allowed. For $2 p$ electrons, the dipole allowed transitions are then $2 p \rightarrow 4 s$ and $2 p \rightarrow 3 d$. Although nondipole transitions occur, it has been shown that the contribution of these transitions becomes significant only several hundred $\mathrm{eV}$ above the $L_{2,3}$ edge onset. ${ }^{27}$ Furthermore, dipole allowed transitions to the $4 s$ states are not expected to contribute significantly, ${ }^{28}$ and we consider only transitions of the type $2 p \rightarrow 3 d$.

Under these assumptions, the measured and normalized intensity is then given by ${ }^{20}$

$$
I \approx \rho^{u}(3 d) \frac{\int_{E_{0}}^{E_{1}}|\langle 3 d|\mathbf{r}| 2 p\rangle|^{2} d \varepsilon}{\int_{E_{0}+50}^{E_{0}+100}|\langle\varepsilon d|\mathbf{r}| 2 p\rangle|^{2} d \varepsilon}=\rho^{u}(3 d) \frac{M_{\text {white line }}}{M_{\text {continum }}} .
$$

Here, $\rho^{u}(3 d)$ is the density of unoccupied TM $3 d$ states and $\varepsilon d$ is a continuum state with energy $\varepsilon$ and angular momentum quantum number $l=2$. The numerator $|\langle 3 d|\mathbf{r}| 2 p\rangle|^{2}$ is the transition probability from the $2 p$ to $3 d$ states, while the denominator is the same for transitions to the continuum. The ratio of the matrix elements $M_{\text {white line }} / M_{\text {continuum }}$ was calculated by Pearson et al. in an earlier work. ${ }^{20}$ The total TM $3 d$ occupancy $n_{3 d}$ can now be obtained through the relation

$$
n_{3 d}=10-\rho^{u}(3 d)=10-I \frac{M_{\text {continuum }}}{M_{\text {white line }}} .
$$

The statistical error in our experiments is small. We report standard deviations in the measured $3 d$ occupancy less than 0.16 electrons/atom, and in some measurements as low as 0.01 electrons/atom. However, a higher uncertainty is associated with the absolute value of the $d$ occupancy. This uncertainty is primarily introduced by the different possible boundary choices for the white-line integration and the method of continuum subtraction. Graetz et al. ${ }^{21}$ estimate that this error is approximately \pm 0.4 electrons/atom. However, the relative errors are small, and given the observed standard deviations, we expect the relative changes in $d$ occupancy we obtain to accurately reflect changes in the electronic structure upon compound formation.

\section{RESULTS}

Normalized spectra from pure Co and the three Co-based skutterudites are shown in Fig. 2. The background subtraction in the case of $\mathrm{CoSb}_{3}$ was complicated by the delayed edge of the $\mathrm{Sb} M_{4,5}$ transitions, which extends several hundred $\mathrm{eV}$ beyond their onset at $528 \mathrm{eV}$. Furthermore, the $\mathrm{Sb}$ $M_{2,3}$ edges at 766 and $812 \mathrm{eV}$ add to the complications of subtracting the background. This causes an apparently protracted onset of the Co $L_{2,3}$ edges for $\mathrm{CoSb}_{3}$, while the other three compounds display a rather sharp onset.

The spectra display a large increase in the white-line intensity of $\mathrm{CoP}_{3}$ and $\mathrm{CoAs}_{3}$ compared to that observed in pure Co. The $L_{3}$ peak of $\mathrm{CoSb}_{3}$ is slightly less intense than the $L_{3}$

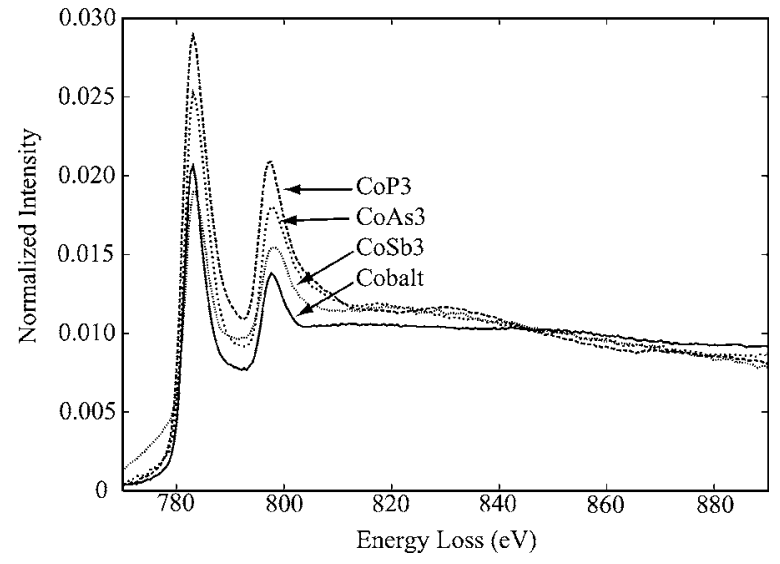

FIG. 2. Normalized EEL spectra of the Co $L_{2,3}$ edge of pure cobalt and the three Co-based skutterudites.

peak of pure cobalt, while the $L_{2}$ peak is more intense. We attribute this difference in ratio to the mentioned problems with background subtraction. The $L_{2,3}$ splitting is approximately $14.5 \mathrm{eV}$ for all the Co-based compounds.

Using the observed white-line intensities and Eq. (3), with the matrix element correction factor given by Pearson et $a l .,{ }^{20}$ we calculate the $3 d$ occupancy of the studied compounds. These are given in Table I. We see that the $3 d$ occupancy of pure Co is 8.3 electrons, suggesting that the elemental cobalt is close to a $3 d^{8} 4 s^{1}$ valence state, consistent with the assumptions made by Pearson et al. In comparison, the Co $3 d$ orbitals of the skutterudites are significantly depleted, displaying occupancies of 7.53, 7.90, and 8.02 electrons for $\mathrm{CoP}_{3}, \mathrm{CoAs}_{3}$, and $\mathrm{CoSb}_{3}$, respectively.

As mentioned above, $\mathrm{Sb} M$ transitions extend beyond the Co $L_{2,3}$ onset. This causes an imperfect fit of the power law to the background below the $L_{2,3}$ peaks. To estimate the effect of this imperfect fit, we apply a power-law background model to a spectrum from pure $\mathrm{Sb}$ (data from the EELS Atlas $^{30}$ ). When fitted to the same $30 \mathrm{eV}$ window used in the analysis of $\mathrm{CoSb}_{3}$, we are left with a nonuniform residual.

TABLE I. Average observed transition metal $3 d$ occupancy $\left(n_{3 d}\right)$ for the pure metals and the skutterudites. We also list the change of occupancy in the skutterudite vs the appropriate pure transition metal $\left(\Delta n_{3 d}\right)$. The final column is the relevant TMpnictogen electronegativity difference $(\Delta \chi)$ in Pauling units (Ref. 29). A negative value signifies the higher electronegativity of the pnictogen relative to the TM.

\begin{tabular}{lccc}
\hline \hline Compound & $\mathrm{TM} n_{3 d}(\sigma)$ & $\Delta n_{3 d}$ & $\Delta \chi$ \\
\hline $\mathrm{Co}$ & $8.30 \pm 0.07$ & \\
$\mathrm{CoP}_{3}$ & $7.53 \pm 0.05$ & -0.77 & -0.31 \\
$\mathrm{CoAs}_{3}$ & $7.90 \pm 0.12$ & -0.40 & -0.30 \\
$\mathrm{CoSb}_{3}$ & $8.02 \pm 0.15$ & -0.28 & -0.17 \\
$\mathrm{CoSb}_{3}$ (with correction) & $7.89 \pm 0.16$ & -0.41 & -0.17 \\
$\mathrm{Ni}_{\mathrm{NiP}}$ & $8.71 \pm 0.01$ & \\
$\mathrm{Fe}$ & $8.59 \pm 0.09$ & -0.13 & -0.28 \\
$\mathrm{LaFe}_{4} \mathrm{P}_{12}$ & $6.87 \pm 0.06$ & \\
\hline \hline
\end{tabular}




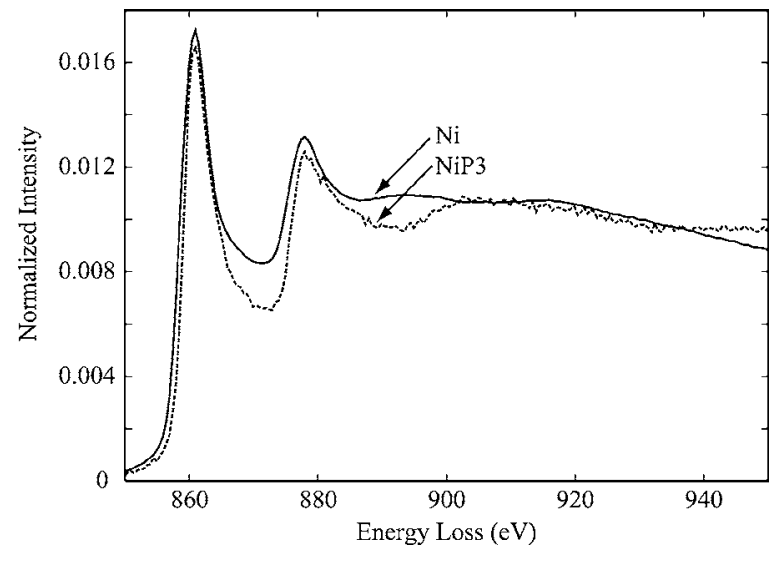

FIG. 3. Normalized spectra from pure nickel and $\mathrm{NiP}_{3}$.

The effect of this residual is to underestimate the white-line intensity relative to the continuum, thereby overestimating the $3 d$ occupancy. To correct this error, we use the data from the EELS Atlas to estimate the relative error in the background fit. This correction is then applied to the $\mathrm{CoSb}_{3}$ data, reducing the $3 d$ occupancy by approximately 0.1 electrons/ atom. Although this correction may be reasonable, it relies on the assumption that the $\mathrm{Sb} M$ edges are unaltered in $\mathrm{CoSb}_{3}$ as compared with the pure $\mathrm{Sb}$. These corrections should therefore be treated with some care. However, we expect the $\mathrm{Sb} 3 d \rightarrow$ continuum transitions to dominate, and the edge will therefore be unaltered in the compound compared to the pure element.

The normalized spectra for $\mathrm{Ni}$ and $\mathrm{NiP}_{3}$ are shown in Fig. 3 . We note that the white-line intensity of these compounds are significantly reduced compared to those of the Co-based compounds, corresponding to a filling of the $3 d$ states due to the extra Ni electron. The $3 d$ occupancy of nickel obtained from our experiments is 8.71 electron/atom, which is fairly close to the expected value with $\mathrm{Ni}$ in a $3 d^{9} 4 s^{1}$ valence state. Although the spectral features of the $\mathrm{NiP}_{3}$ and pure metal seem quite different, the obtained $3 d$ occupancy of the skutterudite is only slightly reduced compared to the pure metal. The $3 d$ occupancy of $\mathrm{NiP}_{3}$ is found to be 8.59 electrons/ atom, a depletion of only 0.13 electrons relative to the pure metal (see Table I).

The most dramatic effect is observed for the white-line intensity of iron in the filled skutterudite $\mathrm{LaFe}_{4} \mathrm{P}_{12}$. Figure 4 shows the normalized spectrum for this compound together with that of pure iron. We immediately note that the whiteline intensity of the pure metal is significantly larger than that observed in the Co- and Ni-based compounds. The $3 d$-occupancy obtained for pure iron is 6.9 electrons/atom, in excellent agreement with the expected $3 d^{7} 4 s^{1}$ valence configuration. In comparison, the $\mathrm{Fe} L_{2,3}$ lines of $\mathrm{LaFe}_{4} \mathrm{P}_{12}$ are significantly decreased, signaling a filling of the $3 d$ states corresponding to +0.77 electrons/atom relative to the pure metal. This filling gives a total $3 d$ occupancy of iron in the skutterudite of 7.66 electrons/atom, close to that observed for cobalt in the binary skutterudites.

\section{DISCUSSION}

The results presented in the previous section suggest significant changes in the local electronic structure of the tran-

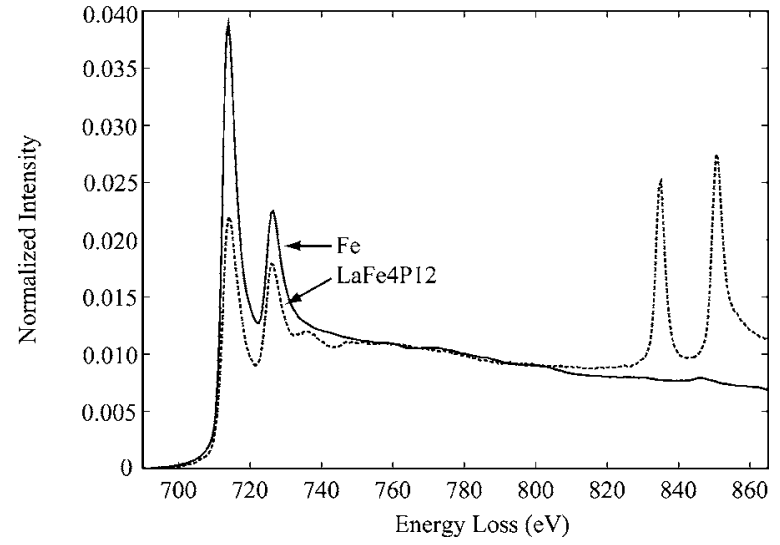

FIG. 4. Normalized spectra from the pure iron and $\mathrm{LaFe}_{4} \mathrm{P}_{12}$ samples. The sharp absorption peaks at high energy side are from the La $M_{4,5}$ transitions.

sition metals upon formation of the binary skutterudites. In the cobalt-based skutterudites, the depletion of the $d$ band ranges from -0.77 to -0.28 electrons/atom. However, these results do not give an indication whether the cause of this depletion is charge transfer from cobalt to the pnictogens or hybridization of outer valence states increasing the amount of $d$ character above the Fermi level. We observe a general correlation between the difference in electronegativity $\Delta \chi$ and the changes in $d$-band occupancy (see Table I). This suggests that there may be an ionic contribution to the bonding of the skutterudites. Furthermore, Anno et al. ${ }^{12}$ found small chemical shifts in the TM $2 p$ binding energy of $\mathrm{CoAs}_{3}$ and $\mathrm{CoSb}_{3}$ which may further indicate charge transfer from Co to the pnictogen, and thus an ionic component to the bonding. Our results are consistent with these findings.

However, the details of our experimental results show a less straightforward relationship. For example, $\Delta \chi$ is very different for $\mathrm{CoAs}_{3}$ and $\mathrm{CoSb}_{3}$, but the observed depletion is virtually the same. Furthermore, only a small depletion is observed in $\mathrm{NiP}_{3}$, even though $\Delta \chi$ is only slightly less than in $\mathrm{CoP}_{3}$. A purely ionic picture based on considerations of electronegativity is therefore not sufficient for a systematic description of bonding in the skutterudites. Indeed, there is ample evidence of strong covalent bonding between the transition metals and pnictogens. First, the difference in electronegativity between the Pn and TM atoms is 0.3 or less. In comparison, other compounds usually considered to exhibit ionic bonding (e.g., $\mathrm{CoO}$ ) display a difference of 1.5 or more. We, therefore, do not expect any significant ionic character in the bonding based on the electronegativity values. Second, the observed TM-Pn bond length is close to the sum of the elemental covalent radii. ${ }^{2} \mathrm{We}$, therefore, expect the TM-Pn bonding in the skutterudites to be mainly covalent in nature, with only a small degree of charge transfer between the elements.

The most frequently used model for covalent bonding in skutterudites is due to Dudkin. ${ }^{31}$ In skutterudites, the transition metal is octahedrally coordinated by pnictogen atoms, giving rise to octahedral $d^{2} s p^{3}$ hybrid orbitals. This allows the transition metal to form strong bonds with the six surrounding pnictogen atoms. The Dudkin model is generally 
confirmed by site- and symmetry-projected density of states from DFT calculations. Typically, a large overlap between the TM $3 d$ and Pn $3 p$ states is observed, ${ }^{7,9,32}$ indicating bonding through hybridization.

The Dudkin model successfully predicts that the binary skutterudites with column 9 transition metals (Co, Rh, and Ir) should be diamagnetic semiconductors. The skutterudite structure does not generally form if the these transition metals are substituted by those of column 8 or 10 . An exception to this is the binary $\mathrm{NiP}_{3}$, which in the Dudkin model has an "excess" of one electron, thereby giving metallic conduction. This is confirmed by experimental ${ }^{33}$ and theoretical ${ }^{6}$ investigations.

Our experiments suggest that this hybridization increases the amount of available $d$-character above the Fermi level for the Co-based skutterudites. This effect is largest in $\mathrm{CoP}_{3}$ where the change from pure cobalt is -0.77 electrons/atom, giving a total $3 d$ occupancy of 7.53 electrons/atom. In comparison, the $d$-band occupancy of $\mathrm{NiP}_{3}$ is only slightly reduced compared to the pure metal, giving a total occupancy of 8.59 electrons. This depletion is far less than what we observe in $\mathrm{CoP}_{3}$, but it is interesting to note that the total occupancy in the nickel-based skutterudite is raised almost exactly one electron/atom above that of the cobalt-based compound. This suggests that the excess electron introduced to the structure is quite accurately described in a rigid band approach.

An experimental comparison with $\mathrm{FeP}_{3}$ is not possible as this compound does not form. In the Dudkin model, it is considered electron deficient, as iron lacks one electron to form the hybrid $d^{2} s p^{3}$ orbitals required to stabilize the transition metal in an octahedron of pnictogens. However, filling the voids in the skutterudite structure with electropositive elements such as La allows the formation of filled skutterudites with iron substituted for cobalt. This is understood in terms of $\mathrm{La}$ in a $3+$ valence state after donating the necessary electrons to saturate the Fe-P bond sufficiently to stabilize the structure. This would entail a transfer of charge away from La into the bonding region between $\mathrm{Fe}$ and $\mathrm{P}$, occupying the hybrid bonding orbitals.

In $\mathrm{LaFe}_{4} \mathrm{P}_{12}$, we observe a filling of states with $\mathrm{Fe} 3 d$ character relative to pure iron. The filling is rather large, corresponding to +0.79 electron/atom giving the greatly reduced $\mathrm{Fe}$ white lines observed in Fig. 4. This filling raises the $d$-band occupancy of iron up to the level observed for cobalt in $\mathrm{CoP}_{3}$, and the two compounds are in a sense locally isoelectronic. Furthermore, if we assume that each $\mathrm{La}$ atom donates three electrons to the four nearest $\mathrm{Fe}$ atoms, this would correspond to a change of +0.75 electrons/iron atom. This is very close to the observed value of +0.79 electrons/ atom and indicates that the charge compensation takes place solely on the Fe sites.

Although the $d$-band occupancies we observe in the skutterudites are consistent with the Dudkin model, further experimental studies and theoretical investigations using bandstructure calculations are needed to understand the bonding mechanisms in the skutterudites. In particular, energy-loss edges related to the pnictogens should be investigated, and changes in the calculated local density of states (DOS) of $\mathrm{Ni}$ in $\mathrm{NiP}_{3}$ and $\mathrm{Fe}$ in $\mathrm{LaFe}_{4} \mathrm{P}_{12}$ should be compared to the experimental results of this work. Also, further experimental studies using EELS with higher-energy resolution would be useful to further investigate hybridization effects in these materials.

\section{CONCLUSION}

We have used electron energy-loss spectroscopy to study the unoccupied density of states of a series of binary skutterudites. These studies reveal a significant increase in the Co $L_{2,3}$ white lines as compared to pure Co metal, indicating an emptying of the Co $3 d$ states upon formation of the skutterudite compounds. This is consistent with the conclusions of Anno et al. ${ }^{12}$ who investigated the occupied states below the Fermi-level, while we have probed the unoccupied states above the Fermi level. Since the Co-Pn bonding in these materials is predominantly covalent as suggested by Dudkin, ${ }^{31}$ we attribute this effect mainly to $d^{2} s p^{3}$ hybridization causing an increase in the number of empty states with $d$ character. In comparison, only a small emptying of $d$ states is observed for $\mathrm{NiP}_{3}$.

Furthermore, our experimental results on $\mathrm{LaFe}_{4} \mathrm{P}_{12}$ indicate a filling of the $\mathrm{Fe} 3 d$ states, allowing iron to form the $d^{2} s p^{3}$ hybrid states needed to stabilize the skutterudite structure. This supports previous models of bonding where charge is transferred from the La atoms to the Fe-P complex and indicates that the charge compensation mainly takes place on the Fe sites.

\section{ACKNOWLEDGMENTS}

The authors would like to acknowledge support from the University of Oslo through the FUNMAT@UiO program and the U.S. DOE through Grant No. DE-FC36-05GO15065. One of the authors (Ø.P.) would also like to thank Shu Miao for help with the TEM and spectrometer during his stay at Caltech.
${ }^{1}$ A. Kjekshus and G. Pedersen, Acta Crystallogr. 14, 1065 (1961).

${ }^{2}$ C. Uher, Semicond. Semimetals 69, 139 (2001).

${ }^{3}$ G. S. Nolas, D. T. Morelli, and T. M. Tritt, Annu. Rev. Mater. Sci. 29, 89 (1999).

${ }^{4}$ G. Chen, M. S. Dresselhaus, G. Dresselhaus, J. P. Fleurial, and T. Caillat, Int. Mater. Rev. 48, 45 (2003).
${ }^{5}$ D. J. Singh and W. E. Pickett, Phys. Rev. B 50, 11235 (1994).

${ }^{6}$ M. Llunell, P. Alemany, S. Alvarez, V. P. Zhukov, and A. Vernes, Phys. Rev. B 53, 10605 (1996).

${ }^{7}$ M. Fornari and D. J. Singh, Phys. Rev. B 59, 9722 (1999).

${ }^{8}$ O. M. Løvvik and Ø. Prytz, Phys. Rev. B 70, 195119 (2004).

${ }^{9} \varnothing$. Prytz, O. M. Løvvik, and J. Taftø, Phys. Rev. B 74, 245109 
(2006).

${ }^{10}$ G. J. Long, B. Mahieu, B. C. Sales, R. P. Hermann, and F. Grandjean, J. Appl. Phys. 92, 7236 (2002).

${ }^{11}$ I. Devos, M. Womes, M. Heilmann, J. Olivier-Fourcade, J. C. Jumas, and J. L. Tirado, J. Mater. Chem. 14, 1759 (2004).

${ }^{12}$ H. Anno, K. Matsubara, T. Caillat, and J. P. Fleurial, Phys. Rev. B 62, 10737 (2000).

${ }^{13}$ I. Lefebvre-Devos, M. Lassalle, X. Wallart, J. Olivier-Fourcade, L. Monconduit, and J. C. Jumas, Phys. Rev. B 63, 125110 (2001).

${ }^{14}$ R. D. Leapman, L. A. Grunes, and P. L. Fejes, Phys. Rev. B 26, 614 (1982)

${ }^{15}$ J. Tafto and O. L. Krivanek, Phys. Rev. Lett. 48, 560 (1982).

${ }^{16}$ T. I. Morrison, M. B. Brodsky, N. J. Zaluzec, and L. R. Sill, Phys. Rev. B 32, 3107 (1985).

${ }^{17}$ R. F. Klie, Y. Zhu, G. Schneider, and J. Tafto, Appl. Phys. Lett. 82, 4316 (2003)

${ }^{18}$ L. A. J. Garvie and P. R. Buseck, Am. Mineral. 89, 485 (2004).

${ }^{19}$ D. H. Pearson, B. Fultz, and C. C. Ahn, Appl. Phys. Lett. 53, 1405 (1988).

${ }^{20}$ D. H. Pearson, C. C. Ahn, and B. Fultz, Phys. Rev. B 47, 8471 (1993).

${ }^{21}$ J. Graetz, C. C. Ahn, H. Ouyang, P. Rez, and B. Fultz, Phys. Rev. B 69, 235103 (2004).
${ }^{22}$ V. J. Keast, A. J. Scott, R. Brydson, D. B. Williams, and J. Bruley, J. Microsc. 203, 135 (2001).

${ }^{23}$ D. H. Pearson, C. C. Ahn, and B. Fultz, Phys. Rev. B 50, 12969 (1994).

${ }^{24}$ H. Ouyang and K. Jen-Tai, J. Appl. Phys. 92, 7510 (2002).

${ }^{25}$ A. Watcharapasorn, R. C. DeMattei, R. S. Feigelson, T. Caillat, A. Borshchevsky, G. J. Snyder, and J. P. Fleurial, J. Appl. Phys. 86, 6213 (1999).

${ }^{26}$ R. F. Egerton, Electron Energy Loss Spectroscopy in the Electron Microscope, 1st ed. (Plenum, New York, 1986).

${ }^{27}$ R. D. Leapman, P. Rez, and D. F. Mayer, J. Chem. Phys. 72, 1232 (1980).

${ }^{28}$ D. A. Muller, D. J. Singh, and J. Silcox, Phys. Rev. B 57, 8181 (1998).

${ }^{29}$ P. W. Atkins, Quanta: A Handbook of Concepts, 2nd ed. (Oxford University Press, Oxford, 1991).

${ }^{30}$ C. C. Ahn and O. L. Krivanek, EELS Atlas (Gatan, Warrendale, 1983).

${ }^{31}$ L. D. Dudkin, Sov. Phys. Tech. Phys. 3, 216 (1958).

${ }^{32}$ K. Koga, K. Akai, K. Oshiro, and M. Matsuura, Phys. Rev. B 71, 155119 (2005).

${ }^{33}$ I. Shirotani, T. Adachi, K. Tachi, S. Todo, K. Nozawa, T. Yagi, and M. Kinoshita, J. Phys. Chem. Solids 57, 211 (1996). 